\title{
Coronary Artery Disease Automatic Classification
}

\author{
Samuel A. Freitas ${ }^{1}$, Cristiano A. da Costa $^{1}$, Gabriel de O. Ramos $^{1}$ \\ ${ }^{1}$ Graduate Program in Applied Computing, Universidade do Vale do Rio dos Sinos - RS - Brazil \\ samuelafeedu.unisinos.br, gdoramos, cacdunisinos.br
}

\begin{abstract}
Atherosclerosis represents the restriction of blood flow in the heart muscle and is one of the main causes of death in the world. The assessment of atherosclerosis is challenging and is currently evaluated by the Fractional Flow Reserve (FFR) and the Quantitative Flow Ratio (QFR). Both exams are based on angiography, which is the gold standard for geometrical assessment. This study presents a pipeline to automatically determine the presence of narrowing in the right coronary artery $(R C A)$ angiography exams, segmenting the artery silhouette, selecting regions of interest (ROIs) followed by a classification model. Initial results suggest a valid sequence of steps to classify the lesion, but require some improvements in the network architecture for better classification accuracy.
\end{abstract}

Resumo. Aterosclerose representa a restrição de fluxo sanguíneo no músculo do coração e é uma das principais causas de morte no mundo. A avaliação de uma aterosclerose é desafiadora e é atualmente realizada pelas técnicas de Fractional Flow Reserve (FFR) e Quantitative Flow Ratio (QFR). Ambos os métodos são baseados em angiografia, que é o padrão-ouro para avaliação geométrica de lesões. Esse estudo apresenta uma cadeia de etapas para a determinação automática da presença de estreitamentos arteriais em angiografias de artéria coronária esquerda (ACE), segmentando a silhueta da artérias, selecionando regiões de interesse seguidas de um modelo classificador. Resultados iniciais indicam a validade da sequência de etapas para classificação, ainda necessitando que melhorias na arquitetura para melhor acurácia.

\section{Introduction}

Cardiovascular diseases (CVD) was the number one cause of death in the world in 2019 [World Health Organization 2020]. CVD includes heart and blood vessels disorders (a.k.a. coronary artery disease - CAD), and are mainly caused by atherosclerosis, which restricts the heart muscle blood flow supply. The blood flow restriction is called ischemia, and it can be associated with chest pain, also known as angina. The relationship between the narrowing and the lesion repercussion can be only achieved with the correct hemodynamic assessment, determined by an initial visual assessment. The visual assessment of angiographies is the gold standard to classify the atherosclerosis significance but is also insufficient to determine the whole stenosis significance [Hideo-Kajita et al. 2019, Benton et al. 2018, Loewe 2019, Johnson et al. 2016].

Coronary arteries system is composed by two main arteries: the left coronary artery (LCA) and the right coronary artery (RCA). The RCA has a less complex anatomy, 


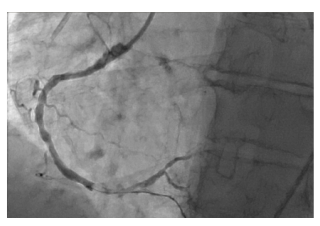

(a)

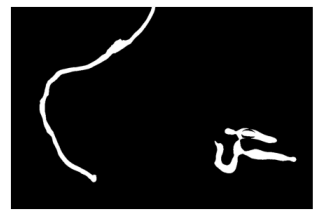

(b)

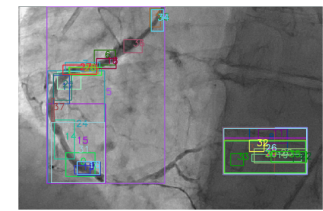

(c)

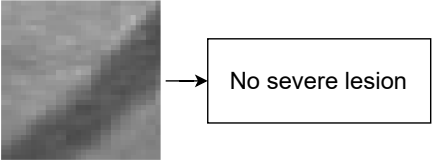

(d)

(e)

Figure 1. In step (a) we have the original exam. In step (b) the original exam segmented together with the artery silhouette highlighted. In step (c) the selective search algorithm applied in the binary silhouette to determine the regions of interest (ROIs). In (d), ROls are then extracted from the original exam. Finally in (e), each segment classified by the CNN.

and consumes around $25 \%$ of the blood flow from aorta [Guyton and Hall 2006]. The stenosis levels in angiographies are classified as: light, with about $50 \%$ of narrowing; moderate, with $50-70 \%$ of narrowing; and severe, with more than $70 \%$ of artery narrowing [Hideo-Kajita et al. 2019, Chakladar et al. 2017]. Severe lesion requires angioplasty, which is a invasive approach to enlarge the arteries and increase the blood flow in the lesion area.

In this work, the lesion classification characteristics are important to the problem definition and the decision of using binary classification. The binary classification proposed intends to determine severe or not severe lesion, once this is the indicator to lead to angioplasty. We present a full pipeline to automatically determine the presence of narrowing in the RCA based on angiography exams. To this end, RCA angiographies are divided into small squares with artery segments to be classified according to the presence or the absence of severe lesions. Emerged methods have achieved accuracy superior to 80\% [Cong et al. 2021, Moon et al. 2021], focused in angiography detection and classification. The main contribution of this study is the segmentation as a preparation module for RCA classification, instead of complete exam evaluation [Moon et al. 2021, Cong et al. 2021]. In this work, the pipeline automates the manual frame determination in the coronary angiography assessment to select the best artery segment candidates for severe lesions screening.

\section{Method}

Our method contains a pipeline composed by three main steps: image pre-processing, selective search, and the binary classification via a Convolutional Neural Network (CNN). Figure 1 represents the complete proposed technique. Our final dataset is composed by two different data. A synthetic and a natural dataset [Antczak and Liberadzki 2018] with 10000 artery segments for both, stenosed and non-stenosed arteries.

The pre-processing step extracts the Regions of interest (ROIs) from the angiography exams and determine the inputs to the classification model. In this step, each exam went through an automatic segmentation, which consists of the frangi filter, followed by adaptative thresholding binarization and then, contours identification with background extraction. This process obtained the complete RCA silhouette and the Figure 1(b) presents a segmented artery example. In some cases, image contrast may vary due to equipment acquisition and it can lead to limitations in the automatic segmentation, requiring man- 

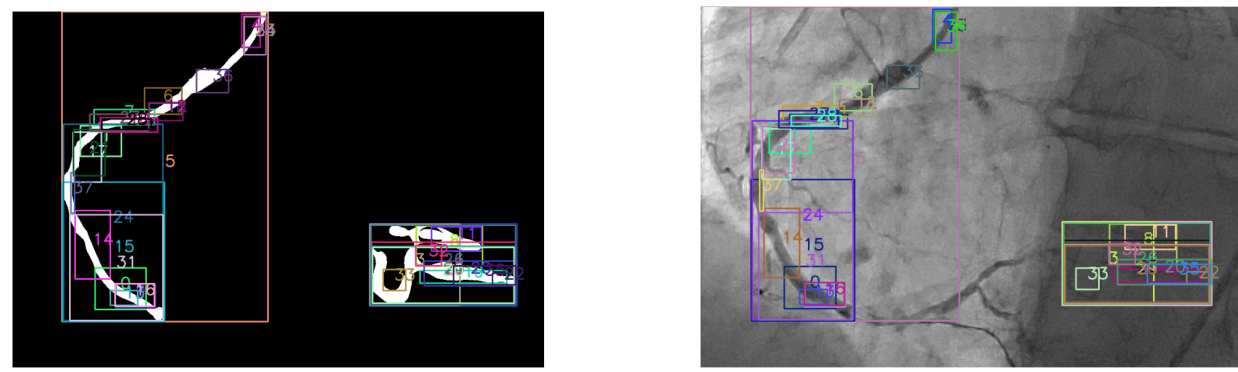

Figure 2. In the top left, the selective search applied to the segmented angiography, reducing the data complexity. In the top right, the ROls applied to the original angiography, which covers the main RCA segment.

ual adjustments in the threshold value for optimal segmentation, affecting the optimal segmentation, but still useful for most of the cases.

The second step, selective search, is known by having a better performance than manual pixel by pixel stride, providing better and less ROIs. The use of segmented image reduced the input complexity to the Region of interest (ROI) search. This choice reduced $98 \%$ of the original ROIs of the exam (from 2700 to 30 ROIs). Figure 1 presents the processing of achieving the regions in segmented image. Figure 1 (c) presents the ROIs search result in the original exam. Selective search provided efficient artery segment suggestions. Then, Figure 1 (d) presents an example of the suggested artery segment. After the selection of segments, all the segments extracted from the exam were resized to the input size of $128 \times 128$ pixels, which matches the CNN architecture input. Filtered RCA segments are then fed in a binary classification CNN in the third and last method step. The third step of the pipeline is a CNN, and three different architectures where evaluated for the angiography context. While, CheXNet is a network pre-trained with medical data, Xception and MobileNet v2 were pre-trained with non-medical datasets. Table 1 presents the best results on each architecture.

\begin{tabular}{lllll}
\hline Base & Learning rate & Final dense layer (neurons) & Accuracy & Loss \\
\hline MobileNetV2 & $1 e-5$ & $100 \times 100 \times 1$ & 0.74 & 0.52 \\
Xception & $1 e-7$ & $100 \times 100 \times 1$ & 0.75 & 0.54 \\
CheXNet & $1 e-4$ & $100 \times 100 \times 100 \times 1$ & 0.76 & 0.63 \\
\hline
\end{tabular}

Table 1. Classification model architecture configurations

\section{Experiments}

The use of transfer learning was decided due to the limited number of samples. Our $\mathrm{CNN}$ is composed by the initial layers of each architecture followed by a binary specific final dense layer with sigmoid activation implemented using TensorFlow. The CNN was trained using a synthetic database [Antczak and Liberadzki 2018] due to its histogram equalization and normalization. As a retrain process, we choose a learning rate varying between $1 e-4$ and $1 e-7$ with binary cross entropy loss function.

\subsection{Numerical Results}

Pre-processing pipeline presented good performance but it depends completely on the segmentation. As shown in Figure 2, areas not completely filtered in the segmentation 
also identify ROIs which can lead to unnecessary evaluation and false positives. The classification into stenosed and non-stenosed arteries achieved the best result with CheXNet architecture and an accuracy of $76 \%$, with a loss of 0.6 . The obtained loss is still high and requires further improvement in future work.

\section{Conclusions and Next Steps}

The pipeline implementation suggests the use of this technique in patient triage, once the fast processing and segmentation reduces the complexity for image classification. Divide responsibility has been shown promising to allow the continuity of the study in the improvement of feature map for coronary angiographies exams. The last part of the pipeline requires further improvements. Currently with an accuracy higher than $70 \%$, some enhancements in the dense layer must be evaluated in order to reach better performance ratios so it can be validate by coronary artery disease (CAD) specialists. Finally, we expected optimize the hyper parameters for better performance.

\section{References}

Antczak, K. and Liberadzki, Ł. (2018). Stenosis Detection with Deep Convolutional Neural Networks. MATEC Web of Conferences, 210:1-6.

Benton, S. M., Tesche, C., De Cecco, C. N., Duguay, T. M., Schoepf, U. J., and Bayer, R. R. (2018). Noninvasive Derivation of Fractional Flow Reserve From Coronary Computed Tomographic Angiography: A Review. Journal of Thoracic Imaging, 33(2):8896.

Chakladar, A., Gan, J., and Edsell, M. (2017). Angiografia arterial coronária. Anaesthesia Tutorial of the Week.

Cong, C., Kato, Y., Vasconcellos, H. D., Ostovaneh, M. R., Lima, J. A., and AmbaleVenkatesh, B. (2021). Deep learning-based end-to-end automated stenosis classification and localization on catheter coronary angiography. medRxiv.

Guyton, A. and Hall, J. (2006). Textbook of Medical Physiology. Elsevier Saunders.

Hideo-Kajita, A., Garcia, H., Schlofmitz, E., and Campos, C. (2019). Atualização sobre tecnologias fisiológicas baseadas em angiografia coronariana - update on coronary angiography-based physiology technologies.

Johnson, N. P., Gould, K. L., Di Carli, M. F., and Taqueti, V. R. (2016). Invasive FFR and Noninvasive CFR in the Evaluation of Ischemia: What Is the Future? Journal of the American College of Cardiology, 67(23):2772-2788.

Loewe, C. (2019). Hemodynamically significant coronary stenosis: Detection with CT Myocardial Perfusion Imaging versus Machine Learning Coronary CT Fractional Flow Reserve. Radiology, 293(2):315-316.

Moon, J. H., Lee, D. Y., Cha, W. C., Chung, M. J., Lee, K.-S., Cho, B. H., and Choi, J. H. (2021). Automatic stenosis recognition from coronary angiography using convolutional neural networks. Computer Methods and Programs in Biomedicine, 198:105819.

World Health Organization, W. (2020). Cardiovascular diseases. https://www.who.97int/news-room/fact-sheets/detail/cardiovascular-diseases-(cvds). 